\title{
Comparison of myocardial scar quantification using phase sensitive inversion recovery (PSIR) versus conventional IR-tLF techniques in patients with ischemic and non-ischemic cardiomyopathy
}

\author{
Heather Warren*, Nowell Fine, Matthew Brymer, Anna MacDonald, \\ Terry Thompson, Frank Prato, Maria Drangova and James White
}

Address: University of Western Ontario, London, ON, Canada

* Corresponding author

from 13th Annual SCMR Scientific Sessions

Phoenix, AZ, USA. 21 -24 January 2010

Published: 21 January 2010

Journal of Cardiovascular Magnetic Resonance 20 I0, I 2(SuppI I):PII5 doi:10.I I86/I532-429X-I2-SI-PII5

This abstract is available from: http://jcmr-online.com/content/I2/SI/PII5

(c) 2010 Warren et al; licensee BioMed Central Ltd.

\section{Introduction}

Delayed enhancement (DE) CMR affords quantitative evaluation of myocardial scar in ischemic and nonischemic populations. Phase correction (PC) of DE optimizes signal intensity differences between normal and abnormal myocardium. However, limited data exists validating it's quantitative assessment of scar using signal thresholding techniques.

\section{Purpose}

To compare scar quantification using PC and un-corrected (UC) DE imaging in a large series of patients.

\section{Methods}

Imaging was performed using a $1.5 \mathrm{~T}$ or $3 \mathrm{~T}$ Siemens scanner in patients with known or suspected cardiomyopathy. Serial short axis DE imaging was performed using a PSIRtFL pulse sequence with the TI time adjusted to optimally null normal myocardium on magnitude images. Semiautomated scar volumes were calculated for matched PC and UC-DE images at 2, 3 and 5 standard deviation (SD) above normal myocardium and compared using a two tailed paired-sample Student's $t$-test. Pearson correlation coefficients were calculated and linear regression analysis performed for PC versus UC methods. Scar border-zone was calculated for the ischemic sub-group using the difference between 3 and 5 SD thresholds and compared in the same fashion.

\section{Results}

119 patients were evaluated. The mean LVEF was $52 \pm$ $21 \%$. A total of 79 patients (66\%) demonstrated abnormal DE, 40 with ischemic scar and 39 with non-ischemic scar (epicardial-based or mid-wall). Linear regression of scar volumes from PC and NC images is shown in Figure 1 (a-c). PC and NC scar volumes for ischemic scar at 2, 3 and 5 SD cut-offs were $36.5 \pm 14.1 \%$ versus $41.0 \pm 15.2 \%$, $27.9 \pm 13.9 \%$ versus $32.4 \pm 15.1 \%$, and $17.8 \pm 12.0 \%$ versus $22.4 \pm 13.8 \%$, respectively ( $\mathrm{p}<0.001$ for all comparisons). A similar relationship was seen for non-ischemic scar at each of the thresholds $(23.0 \pm 14.9 \%$ versus $30.2 \pm$ $15.9 \%, 13.4 \pm 12.5 \%$ versus $19.3 \pm 13.2 \%$, and $5.2 \pm 8.1 \%$ versus $8.8 \pm 9.1 \%$, respectively $(\mathrm{p}<0.001$ for all comparisons)). Pearson correlation coefficients at 2, 3, and 5, SD thresholds were $0.91,0.94$, and 0.96 ( $\mathrm{p}<0.001)$, respectively. For ischemic DE a significant difference was seen in scar border-zone between PC and UC images (8.3 \pm 5.7 and $10.5 \pm 6.1(\mathrm{p}=0.04)$, respectively). The correlation coefficient for scar border-zone was $r=0.69(\mathrm{p}<0.001)$.

\section{Conclusion}

Phase correction of DE images results in significant reductions in quantitative scar volumes at all measured signal thresholds. Quantification of ischemic scar border-zone is similarly reduced using the PSIR sequence due to relatively larger reductions in scar volumes at the lower SD threshold. 

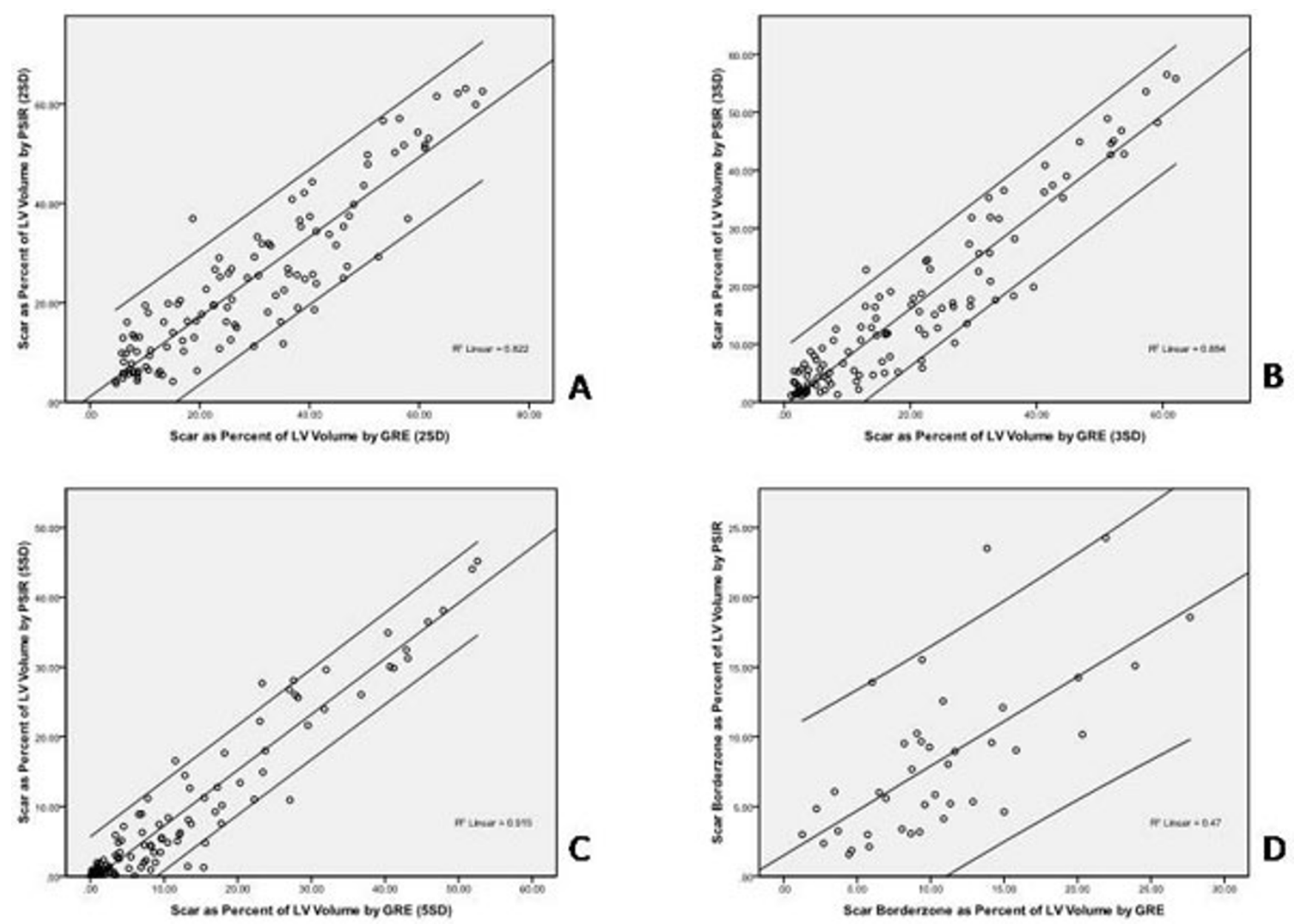

Figure I

(A-D): Scatter plot of percent myocardial scar in entire population (N-I I 9) assessed by standard IR-GRE method ( $x$ axis) and phase corrected (PSIR) method (y axis) using a definition of scar as signal intensity $\geq 2,3$, and 5 standard deviations (SD) above that of normal myocardium (IA, IB, IC, respectively). Panel D depicts volume of scar border-zone in the ischemic subgroup as assessed by both IR-GRE ( $x$ axis) and PSIR ( $y$ axis). Plots include $95 \%$ Confidenec Intervals. 